\title{
Estudio Morfométrico Comparativo entre los Músculos Bíceps Braquial y Braquial con el Objetivo de Establecer el Principal Músculo Flexor del Codo
}

\author{
Comparative Morphometric Study among the Brachial and Brachial Biceps Muscles \\ with Objective of Choosing the Main Muscle of the Flexing of the Elbow \\ "Luiz Carlos Buarque de Gusmão; ** Célio Fernando de Sousa-Rodrigues; \\ ${ }^{* * * *}$ Eduardo José Lopes dos Santos \& ${ }^{* * *}$ Walton Nolasco Araujo Pinto
}

GUSMÃO, L. C. B.; SOUZA-RODRIGUES, F. S. G.; DOS SANTOS, E. J. L. \& PINTO, W. N. A. Estudio morfométrico comparativo entre los músculos bíceps braquial y braquial con el objeto de establecer el principal músculo flexor del codo. Int. J. Morphol., 30(1):185-187, 2012.

RESUMEN: El presente trabajo se propone comparar los diámetros de los vientres del músculo bíceps braquial y músculo braquial con el objeto de esclarecer algunas dudas sobre cuál sería el músculo más potente en la flexión del antebrazo. Las comparaciones fueron realizadas en 50 miembros superiores de cadáveres fijados. Se hizo evidente la diferencia promedio de $0,51 \mathrm{~cm}(5,8 \%)$ en el diámetro del vientre muscular del músculo braquial, ratificando lo encontrado en la revisión bibliográfica, donde el músculo braquial es un importante flexor del codo.

PALABRAS CLAVE: Estudio morfométrico; Músculo bíceps braquial; Músculo braquial; Músculos flexores; Codo.

\section{INTRODUCCIÓN}

Rutinariamente en los gimnasios de musculación, los profesores de Educación Física indican y realizan ejercicios de flexión del antebrazo, enfatizando el objetivo de desarrollar la musculatura del músculo bíceps braquial. Por otro lado, algunos profesores de kinesiología trasmiten a los alumnos que la flexión del codo es realizada principalmente por el músculo bíceps braquial, y casi no citan al músculo braquial ejecutor de esa acción.

La literatura cita que el músculo braquial es el principal flexor del antebrazo (Lippert, 2001; Calais-German, 2002; Behnke, 2004; Rasch, 1991; Palastanga et al., 2001; Hislop \& Montgomery, 1996; Kendall, 1995; Thompson \& Floyd, 2000; Settineri, 1998; Hamill \& Knutzen, 1999; Gusmão \& Lima, 2002; Kisner \& Colby, 1999), siendo por cierto ésta su única función (Behnke). El músculo bíceps braquial, por poseer origen en la escápula, atraviesa dos articulaciones, contribuyendo tanto a la flexión del brazo como a la del antebrazo (Moore, 2001; Gardner et al., 1988).
Parte de la literatura anatómica afirma que el músculo braquial es el "burro de carga" de la articulación del codo (Lippert; Rasch), mientras que el músculo bíceps braquial sería apenas eficiente con el codo en supinación, cuando éste está aproximadamente en $90^{\circ}$ de flexión, perdiendo su eficiencia cuando el codo está extendido (Rasch; Hamill \& Knutzen).

Por otro lado el análisis knesiológico a través de Resonancia Magnética (Tesch, 2001) confirma que los tres mayores flexores del codo son: la cabeza medial del músculo bíceps braquial, la cabeza lateral del bíceps braquial y el músculo braquial, sin embargo, resalta que el músculo bíceps braquial sería más activo en este movimiento.

El presente trabajo se propone comparar los diámetro de los vientres de los músculs citados con el objetivo de dilucidar las dudas de cuál sería el músculo más potente, visto que el medio del vientre muscular es una medida indi-

Profesor associado del Departamento de Morfología de la Universidade Federal de Alagoas; Máster y Doctor en Anatomía Humana, Miembro Titular del Colegio Brasileiro de Cirujanos, Brasil.

** Profesor Adjunto del Departamento de Morfología de la Universidade Federal de Alagoas; Máster y Doctor en Anatomía Humana, Brasil.

**** Monitor de la Disciplina de Anatomía Humana de la Universidade Federal de Alagoas, Brasil.

Trabajo realizado en el Departamento de Morfología de la Universidade Federal de Alagoas - Brasil. 
recta del número de fibras musculares, y éstas sumadas trasmiten la potencia muscular.

\section{MATERIAL Y MÉTODO}

En cumplimiento de lo determinado por la Ley Federal $\mathrm{n}^{\circ} 8.501$, del 30 de noviembre de 1992, y luego de la aprobación institucional fueron utilizados 50 miembros superiores de cadáveres adultos, de ambos sexos, siendo 24 $(48 \%)$ derechos y $26(52 \%)$ izquierdos, pertenecientes al Departamento de Morfología de la Universidad Federal de Alagoas, Brasil.

Para la realización de las medidas, fue utilizado un huincha métrica y un paquímetro. El lugar de la medición del diámetro del vientre muscular fue donde el músculo presentaba la mayor circunferencia.

\section{RESULTADOS}

El promedio del diámetro del músculo braquial fue de $9,36 \mathrm{~cm}$ y del músculo bíceps braquial de $8,85 \mathrm{~cm}$.

Ha resultado evidente una diferencia promedio de $0,51 \mathrm{~cm}(5,8 \%)$ en el diámetro del vientre muscular del músculo braquial con respecto al diámero del músculo bíceps braquial.

$\mathrm{Al}$ analizar los datos de circunferencia de los músculos se ha observado que en 39/50 (78\%) de los miembros estudiados, el músculo braquial presentaba una circunferencia mayor que el músculo bíceps braquial, siendo de 10,2\% el valor promedio de las comparaciones en que el músculo braquial superaba el promedio de las medidas del músculo bíceps braquial.

En 10/50 (20\%) de los miembros estudiados, el músculo bíceps braquial superó en la medida de circunferencia al músculo braquial, siendo de $8,3 \%$ el valor promedio de las medidas en que el músculo bíceps braquial superó el promedio de las medidas del músculo braquial. Solo en un caso 1/50 (2\%) las medidas de los vienres músculares de ambos músculos eran iguales en su diámetro.

Analizando solo las 26 medidas del miembro superior izquierdo, verificamos que el promedio de la circunferencia del músculo braquial era de $8,48 \mathrm{~cm}$ y del músculo braquial de $9,14 \mathrm{~cm}$. Repitiendo el análisis para las medidas del miembro superior derecho, el promedio de circunfe- rencia del músculo bíceps braquial fue de $9,21 \mathrm{~cm}$ y del músculo braquial fue de $9,58 \mathrm{~cm}$.

$\mathrm{Al}$ analizar los datos del miembro superior derecho observamos que 22/24 (91\%) de las medidas, el músculo braquial presentó medida de diámetro mayor. Comparativamente, al examinar los datos del miembro superior izquierdo observamos que 17/26 $(65,4 \%)$ de las medidas el músculo braquial presentó medida de diámetro mayor.

\section{DISCUSIÓN}

Resulta evidente una diferencia promedio mayor de $0,51 \mathrm{~cm}(5,8 \%)$ en el diámetro del vientre muscular del músculo braquial, ratificando lo que relata la literatura.

Cuando analizamos los miembros superiores derecho e izquierdo separadamente verificamos que en el miembro superior derecho se observaba un mayor diametro del músculo braquial. La diferencia promedio del diámetro para el miembro superior izquierdo fue de $0,37 \mathrm{~cm}(4,0 \%)$ mayor para músculo braquial y para el miembro superior derecho de $0,66 \mathrm{~cm}(7,8 \%)$ mayor para el músculo braquial. Las dos observaciones pueden ser justificadas por la mayor predominancia de individuos diestros, reflejándose en un mayor uso del miembro superior derecho y por tanto hipertrofiando más la musculatura del miembo superior derecho. Con relación a la mayor diferencia de circunferencia entre los músculos estudiados (predominio del miembro superior derecho), entendemos que el uso de la musculatura estudiada cuando el individuo es diestro justifica una hipertrofia mayor para el músculo braquial, ya que éste debe ser el principal flexor del antebrazo.

Según estudio de R. M. de Tesch, el uso efectivo de determinado grupo muscular en la flexión del antebrazo en función del tipo de ejercicio ejecutado, se observa mayor participación del músculo braquial en los ejercicios tipo Rosca derecha. Los demás tipos de ejercicios utilizando flexión del codo predomina el grupo muscular del bíceps braquial variando la cabeza medial con la lateral para cada ejercicio ejecutado en el estudio.

Además de las medidas predominantes de circunferencia del músculo braquial, indicando su predominancia en la acción de flexión del codo, está su inserción en la ulna (hueso que otorga la estructura mecánica al antebrazo) y del otro lado está la inserción del músculo bíceps braquial en el radio (hueso accesorio del antebrazo), o sea, el músculo braquial por estar insertado en la ulna, consigue trasmitir efectivamente su potencia para la flexión del codo. 
GUSMÃO, L. C. B.; SOUZA-RODRIGUES, F. S. G.; DOS SANTOS, E. J. L. \& PINTO, W. N. A. Estudio morfométrico comparativo entre los músculos bíceps braquial y braquial con el objeto de establecer el principal músculo flexor del codo. Int. J. Morphol., 30(I):185-187, 2012.

Otro hecho que contribuye con la tesis propuesta en este trabajo está relacionado con las acciones del músculo bíceps braquial, siendo flexor del brazo y antebrazo y participando de la abducción del brazo. En contrapartida el músculo braquial desempeña su única función de flexor del antebrazo, por eso que es llamado "burro de carga" o "caballito flexor".

El origen del músculo bíceps braquial está en la escápula (cabezas lateral y medial) pasando por dos articulaciones (humeral y cubital), de esta forma su vector resultan- te de fuerza no está en la misma dirección de la flexión del codo, diferentemente del músculo braquial que posee el vector resultante de la fuerza de tracción en la misma dirección de la flexión del codo.

Partiendo de la base que la fuerza muscular es proporcional al número de fibras musculares y a su diámetro, y verificado que el músculo braquial tiene mayor diámetro en su vientre muscular, se justifica su importancia en la flexión del antebrazo independiente de la posición de supinación o pronación.

GUSMÃO, L. C. B.; SOUZA-RODRIGUES, F. S. G.; DOS SANTOS, E. J. L. \& PINTO, W. N. A. Comparative morphometric study among the brachial and brachial biceps muscles with objective of choosing the main muscle of the flexing of the elbow. Int. J. Morphol., 30(1):185-187, 2012.

SUMMARY: The present work seeks to compare the diameter of the wombs of the muscle brachial biceps and $\mathrm{m}$. brachial in the intention of settling the doubts on which would be the most potent muscle in the flexing of the forearm. The measures were accomplished in 50 superior members of fastened corpses. A medium difference of $0.51 \mathrm{~cm}(5.8 \%)$ was evident in the diameter of the muscular womb the largest for $\mathrm{m}$. brachial, ratifying what was found in the bibliographical revision, that brachial muscle is an important flexor of the elbow.

KEY WORDS: Morphometric study; Brachial muscle; Brachial biceps muscle; Flexing muscles; Elbow.

\section{REFERENCIAS BIBLIOGRÁFICAS}

Behnke, R. S. Anatomia do movimento. Rio de Janeiro, Artmed, 2004.

Calais-German, B. Anatomia para o movimento: introdução à análise das técnicas corporais. $1^{\mathrm{a}}$ Ed. São Paulo, Manole, 2002.

Gardner, E.; Gray, D. J. \& O'Rahilly, R. Anatomia. Rio de Janeiro, Guanabara Koogan, 1988.

Gusmão, L. C. B. \& Lima, J. S. B. Anatomia Humana (Aplicação aos cursos de educação física e fisioterapia). Alagoas, Ed. Catavento, 2002.

Hamill, J. \& Knutzen, K. M. Bases biomecânicas do movimento humano. Rio de Janeiro, Manole, 1999.

Hislop, H. J. \& Montgomery, J. Provas de função muscular: Técnicas de exame manual. $6^{a}$. ed. São Paulo, Guanabara Koogan, 1996.

Kendall, F. P.; McCreary, E. K. \& Provance, P. G. Músculos Provas e funções: Com postura e dor. $4^{\mathrm{a}} \mathrm{ed}$. Rio de Janeiro, Manole, 1995.

Kisner, C. \& Colby, L. A. Execícios Terapêuticos (Fundamentos e técnicas). $3^{\text {a }}$ ed. Rio de Janeiro, Manole, 1999.

Lippert, L. S. Cinesiologia Clínica para fisioterapeutas. $3^{\text {a }}$ Ed. São Paulo, Guanabara Koogan, 2001.
Moore, K. L. Anatomia Orientada para a clínica. Rio de janeiro, Guanabara Koogan, 2001.

Palastanga, N.; Field, D. \& Soames, R. Anatomia e movimento humano: estrutura e função. $3^{\mathrm{a}}$ ed. São Paulo, Manole, 2001.

Rasch, P. J. Cinesiologia e anatomia aplicada. $7^{\text {a }}$ ed. Rio de Janeiro, Guanabara Koogan, 1991.

Settineri, L. I. C. Biomecânica Noções Gerais. São Paulo, Atheneu, 1988.

Tesch, P. A. Análise cinesiológica através da ressonância magnética. São Paulo, Revinter, 2001.

Thompson, C. W. \& Floyd, R. T. Manual de Cinesiologia estrutural. $12^{\mathrm{a}}$ ed. Rio de Janeiro, Manole, 2000.

Dirección para correspondencia:

Luiz Carlos Buarque de Gusmão

Condomínio Aldebaran Alfa, Cuadra F, n. 08.

Tabuleiro dos Martins.

Maceió-AL.

CEP: 57080-900.

BRAZIL

Recibido : 12-07-2011

Aceptado: 22-10-2011 